\title{
Teste da Hipótese Size-Grain: Influência da Rugosidade do Ambiente sobre Formigas (Hymenoptera: Formicidae)
}

\author{
Luciano Martins ${ }^{\dagger}$, Leandro Xavier Teixeira Chevalier1 \& Fábio Souto Almeida²
}

†In Memorian. 1. Programa de Pós-Graduação em Biologia Animal/ IB/UFRRJ, e-mail: leandrochevalierufrrj@yahoo.com.br. 2. Programa de Pós-Graduação em Ciências Ambientais e Florestais; Departamento de Ciências Administrativas e do Ambiente/ITR/UFRRJ, Três Rios-RJ, Brasil, e-mail: fbio_almeida@yahoo.com.br (Autor para correspondência ${ }^{\bowtie}$ )

EntomoBrasilis 4 (3): 100-102 (2011)

Resumo. Este trabalho objetivou testar a hipótese de que formigas com menor tamanho corporal têm maior sucesso em explorar os interstícios do ambiente, enquanto que ambientes menos rugosos favorecem formigas com tamanho maior. O experimento foi realizado em uma floresta secundária situada no distrito de Ilha Grande, Angra dos Reis-RJ ( $\left.23^{\circ} 11^{\prime} 01^{\prime \prime S}, 44^{\circ} 11^{\prime} 44^{\prime \prime W}\right)$. Foram escolhidos ao acaso 14 locais distantes 10 metros entre si. Em cada local foram demarcadas três parcelas de $50 \mathrm{~cm}$ x $50 \mathrm{~cm}$ com espaçamento de $10 \mathrm{~cm}$ entre si. A serapilheira foi removida das parcelas e aplicou-se tratamentos que simularam ambientes com diferentes níveis de rugosidade: baixo (sem serapilheira); médio (somente folhas de bambu); e alto (com serapilheira). Iscas de sardinha e solução de água com açúcar foram colocadas no centro de cada parcela e a primeira formiga que alcançou as iscas foi coletada. O comprimento total do corpo das formigas coletadas foi significativamente menor no tratamento com maior nível de rugosidade (Teste de Tukey, $\mathrm{p}<$ 0,05). Os resultados confirmaram a hipótese de que formigas menores são favorecidas em ambientes com alto nível de rugosidade.

Palavras-chave: Formicidae; macroecologia; serapilheira.

\section{Testing the Size-grain Hypothesis: Influence of the Environmental Rugosity on Ants (Hymenoptera: Formicidae)}

Abstract. This study aimed to test the hypothesis that smaller ants are more successful in exploring environmental interstices than larger ants, whereas environments with less rugosity are better for larger ants. The experiment was conducted in a secondary forest located in Angra dos Reis, Rio de Janeiro State (23 11'oo"S, 44 11'44”O). Fourteen sites were randomly selected and in each site three plots $(50 \mathrm{~cm} \times 50 \mathrm{~cm})$ were demarcated. The leaf litter was removed from the plots and were applied treatments that simulated environments with different rugosity levels: low (no leaf litter), medium (only bamboo leaves) and high (with leaf litter). Food baits were exposed in the center of the plots and the first ant that access the baits was collected. The total length of the ants was significantly lower in the treatment with higher level of rugosity (Tukey, $\mathrm{p}<0.05$ ). The results confirmed the hypothesis that environments with high levels of rugosity favours smaller ants.

Keywords: Formicidae; leaf litter; macroecology.

D urante o processo evolutivo os organismos são selecionados para os ambientes em função de suas características morfológicas (BEGON et al. 1990; DYER 1995; JANZEN 1969). Segundo a hipótese size-grain, conforme decresce o tamanho de um organismo terrestre que caminha, na sua percepção, seu ambiente torna-se mais rugoso, ou seja, menos plano (KaSPARI \& WeISER 1999; Kaspari \& WeISER 2007). Desse modo, as vantagens de ter pernas longas, mais eficientes para se movimentar de forma veloz sobre um ambiente plano, podem diminuir conforme diminui o tamanho do corpo, enquanto que os custos de se ter pernas longas são acentuados, pois torna limitado o acesso aos interstícios do ambiente, que podem conter recursos alimentares e refúgio (Hölldobler \& Wilson 1990; Kaspari \& WEISER 1999). Assim, a seleção natural pode favorecer pernas proporcionalmente menores com o decréscimo do tamanho do corpo, o que proporciona vantagem competitiva a organismos pequenos em ambientes rugosos (FARJI-BRENER et al. 2004).

Dentre os insetos, as formigas se destacam como organismos abundantes e com elevado número de espécies, além de possuírem expressiva distribuição geográfica, habitando quase todos os ecossistemas terrestres (HÖLLDOBLER \& WILSON 1990). Estudando a morfologia de espécies de formigas, alguns autores encontraram evidências que apoiam a hipótese size-grain (KASPARI \& WeISER 1999; FARJI-BRENer et al. 2004; ESPADAlER \& Gomez 2001). Assim, pode-se supor que formigas de menor tamanho corporal sejam favorecidas em ambientes mais rugosos.

Em relação às formigas que habitam as florestas tropicais, a serapilheira é um componente que está diretamente ligado a rugosidade do ambiente. Desse modo, é possível que formigas de menor tamanho corporal sejam favorecidas em ambientes com maior quantidade de folhedo ou com serapilheira mais heterogênea (KASPARI \& WEISER 2007).

O trabalho objetivou testar a hipótese de que ambientes mais rugosos favorecem formigas com menor tamanho corporal, enquanto que ambientes menos rugosos favorecem formigas com tamanho maior. 


\section{MATERIAL E MÉTODOS}

A coleta de dados foi realizada no mês de novembro de 2009, em uma floresta secundária situada no distrito de Ilha Grande, Angra dos Reis-RJ ( $23^{\circ} 11^{\prime} \mathrm{O} 1^{\prime}$ 'S, $44^{\circ} 11^{\prime} 44^{\prime}$ W). Segundo a classificação de Köppen, o clima da região caracteriza-se como Af (tropical úmido), sem estação seca definida e com precipitação anual média de $2.300 \mathrm{~mm}$ (СHADA et al. 2004).

Foram escolhidos ao acaso 14 locais distantes no mínimo 10 metros entre si. Em cada local foram demarcadas três parcelas de $50 \mathrm{~cm} \times 50 \mathrm{~cm}$ separadas $10 \mathrm{~cm}$ entre si, totalizando 42 parcelas. A serapilheira foi removida das parcelas e aplicouse tratamentos que visavam simular ambientes com diferentes níveis de rugosidade, são eles: baixo (sem serapilheira); médio (somente folhas de bambu); e alto (com serapilheira) (FARJIBRENER et al. 2004) (Figura 1). As folhas de bambu e a serapilheira em geral, que foram coletadas próximo ao local do experimento, foram vistoriadas para se retirar as formigas e o volume utilizado desses materiais foi o mesmo em todas as parcelas $\left(3 \mathrm{dm}^{3}\right)$. Iscas de sardinha e solução de água com açúcar foram colocadas no centro de cada parcela, sobre uma folha seca.

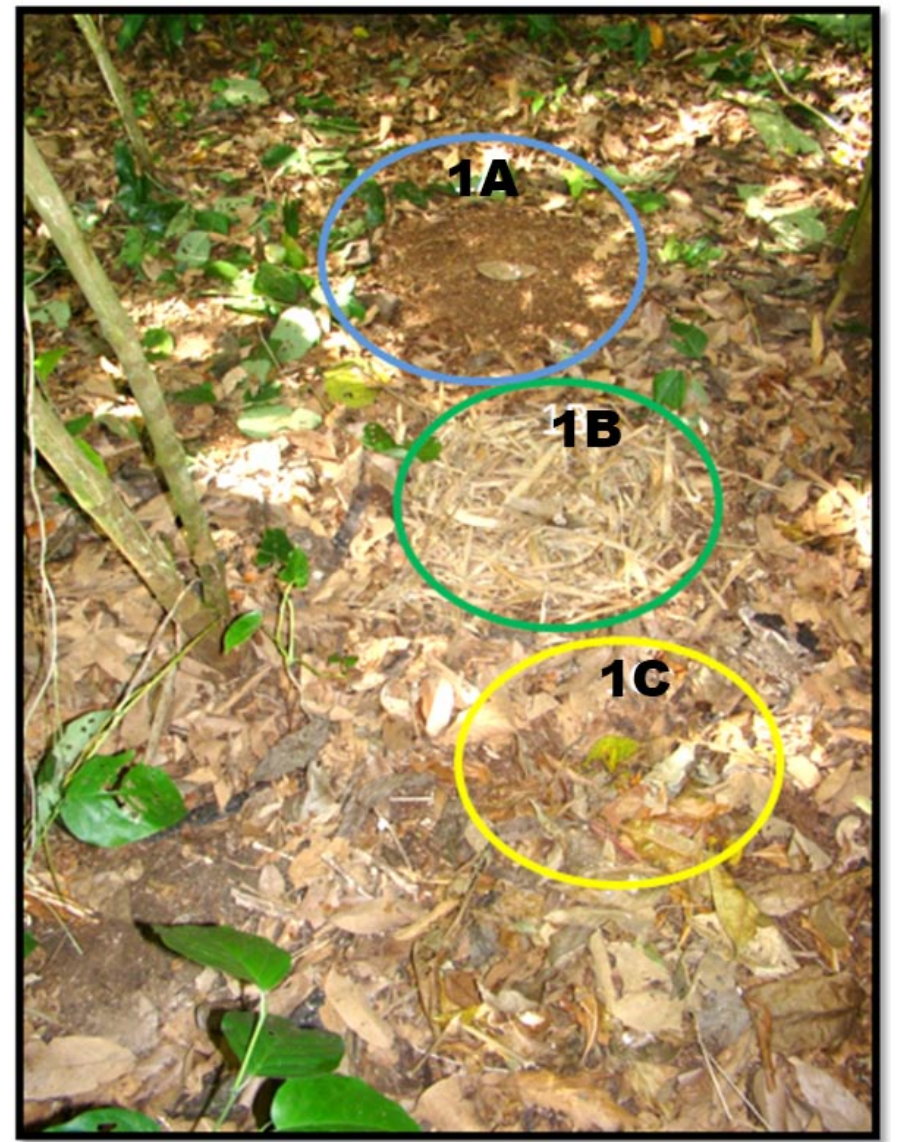

Figura 1. Tratamentos utilizados: (1A) baixo nível de rugosidade (sem serapilheira); (1B) nível médio de rugosidade (folhas de bambu); (1C) alto nível de rugosidade (serapilheira).

Em cada parcela, foi coletada a primeira formiga que chegou à isca e tomou-se a medida do comprimento total do corpo (exceto antenas) de cada formiga. Estas foram ainda identificadas ao nível de gênero. Todas as parcelas foram colonizadas por formigas.

Para análise estatística foi utilizada a análise de variância (ANOVA) e posteriormente o teste de Tukey, a 5\% de probabilidade.

\section{RESULTADOS E DISCUSSÃO}

Foram coletadas formigas pertencentes a sete gêneros, sendo que Camponotus, Crematogaster e Pachycondyla ocorreram principalmente nas parcelas com menor nível de rugosidade e formigas dos gêneros Pheidole e Solenopsis, formigas que apresentaram os menores valores de comprimento do corpo, foram coletadas principalmente nas parcelas com maior nível de rugosidade (Tabela 1).

Tabela 1. Número de formigas, por gênero, coletadas em parcelas com diferentes níveis de rugosidade do ambiente e o comprimento médio do corpo.

\begin{tabular}{|c|c|c|c|c|}
\hline \multirow{2}{*}{ Gênero } & \multicolumn{3}{|c|}{ Nível de Rugosidade } & \multirow{2}{*}{$\frac{\text { Comprimento }}{\text { (cm) }}$} \\
\hline & Baixo & Médio & Alto & \\
\hline Camponotus & 3 & 1 & 1 & 0,62 \\
\hline Crematogaster & 3 & 1 & 2 & 0,41 \\
\hline Labidus & o & 1 & o & 0,45 \\
\hline Odontomachus & 1 & 5 & o & 0,91 \\
\hline Pachycondyla & 4 & 3 & 1 & 1,41 \\
\hline Pheidole & 3 & 2 & 6 & 0,24 \\
\hline Solenopsis & o & 1 & 4 & 0,18 \\
\hline
\end{tabular}

O comprimento total do corpo das formigas coletadas foi significativamente menor no tratamento com maior nível de rugosidade (Figura 2). Os resultados confirmaram a hipótese de que formigas menores são favorecidas em ambientes com alto nível de rugosidade. Já em ambientes planares, as formigas maiores podem ser favorecidas. Resultados similares foram encontrados por outros autores, pois observaram que as formigas que primeiro alcançaram as iscas nos ambientes mais rugosos foram cerca de $40 \%$ menores que os indivíduos que primeiro alcançaram as iscas em ambientes planos (FARJI-BRENER et al. 2004). Todavia, outros autores não encontraram suporte para a hipótese size-grain, pois constataram que classes de tamanho de corpo menores não foram mais específicas em ambientes rugosos que em planos e formigas pequenas foram mais abundantes em ambientes planares (PARR et al. 2003).

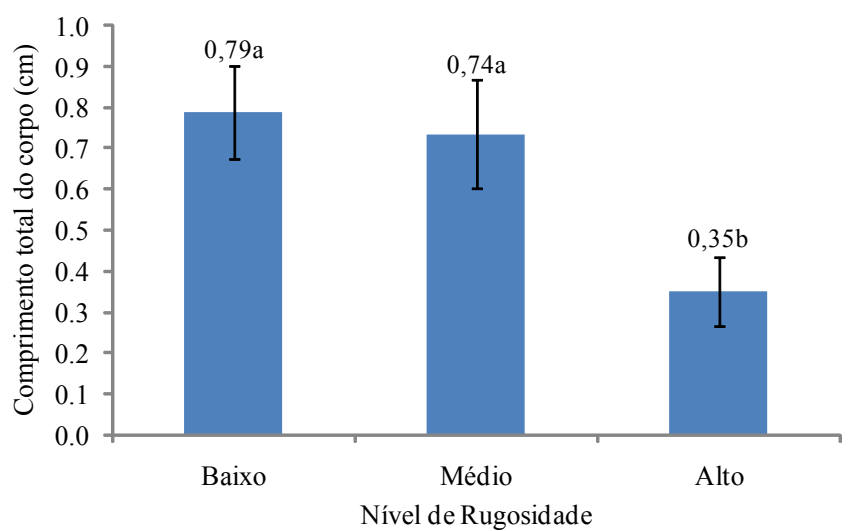

Figura 2. Comprimento total do corpo (média \pm EP) de formigas que chegaram primeiro às iscas em parcelas com diferentes níveis de rugosidade. Letras diferentes indicam diferença significativa pelo teste de Tukey a $5 \%$ de probabilidade.

No presente trabalho também foi constatado que a homogeneidade da serapilheira de folhas de bambu pode ter funcionado como um ambiente de baixo nível de rugosidade. Isso demonstra que não só a quantidade de serapilheira é importante para caracterizar o nível de rugosidade, também a heterogeneidade é importante (KASPARI \& WEISER 2007).

O que emerge desses resultados é que a rugosidade do terreno pode influenciar a distribuição das espécies de formigas e, assim, toda a sua comunidade (SARTY et al. 2006). Espécies polimórficas, por exemplo, podem ter vantagem competitiva, pois, por possuírem operárias com diferentes tamanhos, podem forragear com sucesso em ambientes com diferentes níveis de rugosidade.

Os resultados confirmam a hipótese de que formigas menores são favorecidas em ambientes com alto nível de rugosidade. Já em ambientes planares, as formigas maiores são favorecidas. A rugosidade do terreno pode influenciar a distribuição das espécies e, desse modo, toda a comunidade de 
formigas.

\section{AGRADECIMENTOS}

A Coordenação de Aperfeiçoamento de Pessoal de Nível Superior (CAPES) pela bolsa concedida ao primeiro autor. A Antônio José Mayhé-Nunes, Alejandro G. Farji-Brener e Jarbas Marçal de Queiroz, professores da disciplina de Ecologia de Formigas da UFRRJ, durante a qual os dados do presente estudo foram coletados. A UERJ pela estadia em sua base em Ilha Grande.

\section{REFERÊNCIAS}

Begon, M., J.L. Harper \& C.R. Townsend, 1990. Ecology: individuals, populations and communities. Tauton, Blackwell Science, 945p.

Dyer, L.A., 1995. Tasty generalists and nasty specialists? Antipredator mechanisms in tropical lepidopteran larvae. Ecology, 76: 1483-1496.

Janzen, D.H., 1969. Seed-eaters versus seed size, number, toxicity and dispersal. Evolution, 23: 1-27.

Hölldobler, B. \& E. O. Wilson, 1990. The Ants. Cambridge, The Belknap Press of Harvard University, $732 \mathrm{p}$.

Kaspari, M. \& M.D. Weiser, 1999. The size-grain hypothesis and interspecific scaling in ants. Functional Ecology, 13: 530538 .

\section{Como citar este artigo:}

Martins, L., L.X.T. Chevalier \& F.S. Almeida, 2011. Teste da Hipótese Size-Grain: Influência da Rugosidade do Ambiente Sobre Formigas (Hymenoptera: Formicidae). EntomoBrasilis, 4(3): 100-102. www.periodico.ebras.bio.br/ojs
Kaspari, M. \& M. D. Weiser, 2007. The size-grain hypothesis: do macroarthropods see a fractal world? Ecological Entomology, 32: $279-282$.

Farji-Brener, A.G., G. Barrantes \& A. Ruggiero, 2004. Environmental rugosity, body size and access to food: a test of the size-grain hypothesis in tropical litter ants. Oikos, 104: 165-171.

Espadaler, X. \& C. Gomez, 2001. Formicine ants comply with the size-grais hypothesis. Functional Ecology, 15: 136-139.

Chada, S.S., E.F.C. Campello \& S.M. Faria, 2004. Sucessão vegetal em uma encosta reflorestada com leguminosas arbóreas em Angra dos Reis, RJ. Revista Árvore, 28: 801-809.

Parr, Z.J.E, C.L. Parr \& S.L. Chown, 2003. The size-grain hypothesis: a phylogenetic and field test. Ecological Entomology, 28: 475-481.

Sarty, M., K.L. Abbott \& P.J. Lester, 2006. Habitat complexity facilitates coexistence in a tropical ant community. Oecologia, 149: 465-473.

Recebido em: 01/01/2011

Aceito em: 31/o8/2011
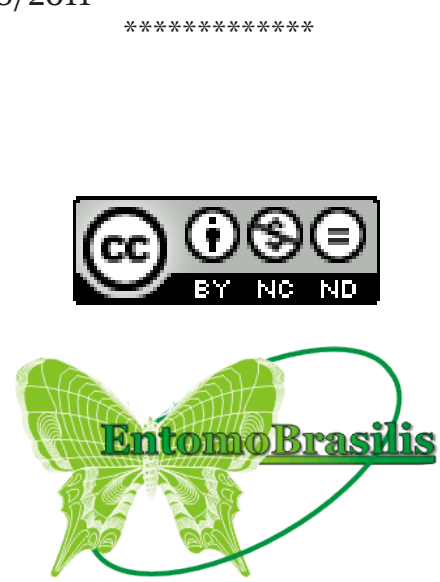

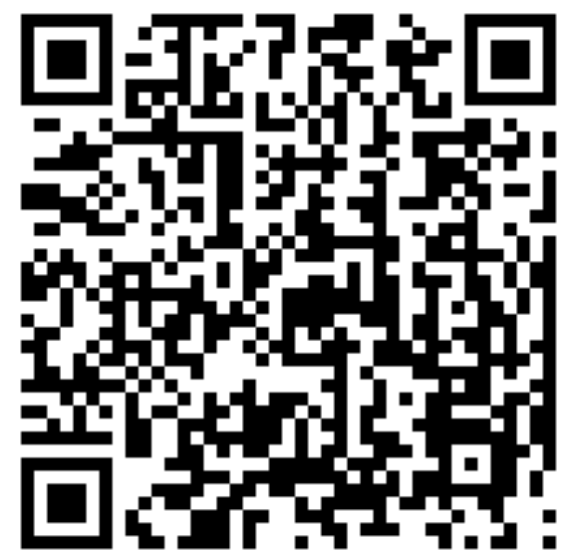

